\title{
A direct proof of improved biased random walk with gastric cancer dataset
}

\begin{abstract}
The relationship between the weight of a single genes and the connection between the genes had been studied. Chemistry and physical sciences have proved the attraction between molecules. Molecules are attracted to each other by bond. This paper is presented to disclose the relationship between weight and connectivity of nodes with biased random walk. An equation of biased random walk which named as significant directed random walk is formed to enhance the connectivity of nodes in directed graph via weigh. To be completely biased to the random walk, references data is implement as directed graph. Weight of genes will be used as one of the parameter in the formula. While the adjacency matrix is further enhanced by Warshall's algorithm to increases the accessibility of nodes via vector. The evolution of random walk is disclosed in this paper as well. Significant directed random walk will be used to prove the importance of weight in this paper. Comparison of the result between biased random walk is presented to prove the enhancement of random walk.
\end{abstract}

Keyword: Biased random walk; Cancer classification; Gene expression dataset 